\title{
Magnetoplasma excitations of two vertically coupled dots
}

\author{
B. Partoenst, A. Matulis \\ Departement Natuurkunde, Universiteit Antwerpen (UIA), \\ Universiteitsplein 1, B-2610 Antwerpen, Belgium
}

(February 19, 2018)

\begin{abstract}
A classical hydrodynamic approach is used to calculate the magnetoplasma excitations of two vertically coupled electron dots. The electrons are confined by different parabolic potentials in which case Kohn's theorem is no longer valid. The equilibrium density profiles of the electrons in both dots are calculated as function of the interdot distance. We find that for unequal confinements of the two dots the electron density in one of the dots becomes ring-like. The electron densities are then used to obtain the magnetoplasma frequencies. The oscillator strengths are calculated, and we find that other then the center of mass modes can be excited due to the electron-electron interactions.
\end{abstract}

PACS numbers: 73.20.Dx, 36.40.Gk 


\section{INTRODUCTION}

Modern semiconductor technology makes it possible to fabricate low-dimensional nanostructures with controllable chemical composition and geometric structure. In artificial atoms a number of electrons are confined into a single quasi-two-dimensional quantum dot which has been widely studied over the past few years (see, for instance, Ref. 1). Most of the work has focused on quantum dots with a soft lateral confinement potential in the heterostructure plane (2D-plane) which are the so called parabolic quantum dots. Such quantum dots exhibit a far-infrared absorption spectrum which reflects only the center of mass motion of the electron system which is a consequence of a generalized Kohn's theorem. 1 When a magnetic field is applied perpendicular to the plane of the quantum dot it results into two absorption peaks whose positions are independent of the number of electrons in the dot. Note that the same absorption spectrum follows from a pure classical analysis. In order to reveal effects due to electron-electron interaction the confinement potential should be anharmonic, or band non-parabolicity effects should be taken into account.

The other possibility to trace the influence of electron-electron interaction on the quantum dot properties is to consider the more sophisticated system of coupled quantum dots. An example of such a system are two vertically coupled dots. The properties of such a system for small number of electrons were studied previously via exact diagonalization 3 or for a model system with an exactly solvable interaction 1 where the main attention was on electron correlations in the ground state, magic numbers and the possibility of the appearance of new type of entangled electronic states. In Ref. 5 it was shown that in the case of a double dot system, containing three electrons, with different parabolic confinement potentials, the electron correlation effects exhibit themselves in the absorption energy. The classical double dot system containing a finite number of electrons exhibits a rich variety of first, second and higher order transitions 6 Two vertically aligned dots is the finite system analog of the well studied bilayer system, 7 which have been shown to exhibit much richer physical phenomena then 2D and 3D systems.

Such coupled dot systems can be realized for example in vertically etched double quantum well structures, ${ }^{1}$ parallel layers of self assembled quantum dots and by using a conducting atomic force microscope tip above a quantum well which can induce locally a dot structure in the quantum well and one beneath the lowest barrier 10 Recently, the successful growth of vertically aligned dots of up to 10 layers of InAs islands separated by GaAs spacer layers were reported.11 These experimental attempts are still in their infancy and to our knowledge no magneto-absorption experiments have been performed yet.

The purpose of the present paper is to study the properties of the system consisting of two vertically coupled quantum dots in a uniform perpendicular magnetic field. We calculate the electron density profiles, the spectrum of magnetoplasma excitations and the corresponding power absorption. We restrict our consideration to dots with a large number of electrons. In this case the classical hydrodynamic approach is adequate, and we follow the ideas presented in the paper of Ye and Zaremba 12 where the single quantum dot with quartic confining potential corrections was considered. A similar technique was used to study one layer electron dots with parabolic 13,14 and non-circular 15 confinement potentials. 


\section{MODEL}

We consider two infinitely thin two-dimensional circular symmetric dots which are vertically aligned and are separated by a distance $a$ as is schematically shown in Fig. 1. The electrons are confined by different parabolic potentials. In the hydrodynamic approach the electrons are described by density functions $\rho_{j}(\mathbf{r})$ where $j=1,2$ refers to the corresponding dot, and $\mathbf{r}=(x, y)$ is the position coordinate in the plane of the dot. Those electron densities create an electrostatic potential $\varphi(\mathbf{r}, z)$ which obeys Poisson's equation

$$
\nabla^{2} \varphi=\frac{4 \pi e}{\epsilon}\left\{\rho_{1}(\mathbf{r}) \delta(z)+\rho_{2}(\mathbf{r}) \delta(z-a)\right\}
$$

where $\epsilon$ is the dielectric constant of the medium the electrons are moving in. We assume that $\epsilon$ is constant thrroughout the whole system which is a good approximation for e.g. the GaAs $/ \mathrm{Al}_{x} \mathrm{Ga}_{1-x}$ As system.

In a purely classical approach, the electrons in both dots achieve an equilibrium distribution in which the lateral force arising from the confining potentials is balanced by the electrostatic repulsive force due to the electrons. Equivalently, the equilibrium conditions can be expressed as the constancy of the chemical potential in each of the dots given by the expressions

$$
\begin{aligned}
& \mu_{1}=\frac{k_{1}}{2} r^{2}-\left.e \varphi(\mathbf{r}, z)\right|_{z=0}, \\
& \mu_{2}=\frac{k_{2}}{2} r^{2}-\left.e \varphi(\mathbf{r}, z)\right|_{z=a} .
\end{aligned}
$$

Here the strength of the parabolic confinement potential is characterized by the corresponding factor $k_{i}$ which is related to the confinement frequency as $\omega_{j}=\sqrt{k_{j} / m}$. Here and further the symbol $m$ stands for the effective electron mass. The difference in the above chemical potentials can be controlled by applying some external bias potential

$$
e V=\mu_{1}-\mu_{2}
$$

In equilibrium the electron densities are circular symmetric $\rho_{j}(\mathbf{r})=\rho_{j}(r)$ and vanish outside some radius $R_{j}$ which must be calculated self-consistently. Those radii are related to the number of electrons in the dots

$$
2 \pi \int_{0}^{R_{j}} \rho_{j}(r) r d r=N_{j}
$$

Condition (2.3) together with definitions (2.2) and Poisson's equation (2.1) form the basic set of equations from which the equilibrium electron density profiles in the dots can be obtained. In a classical description the equilibrium electron distributions are not influenced by an external magnetic field.

In order to obtain the excitation spectrum and the power absorption in the coupled dot system the small harmonic deviations from the electron equilibrium density and potential functions have to be introduced

$$
\delta \rho_{j}(\mathbf{r}), \delta \varphi(\mathbf{r}) \sim e^{i \omega t}
$$


Those additional quantities obey a set of dynamic equations. They are composed of the same Poisson equation (2.1) for the above deviations and the continuity equations

$$
i \omega e \delta \rho_{j}(\mathbf{r})+\nabla \cdot \mathbf{j}_{j}(\mathbf{r})=0
$$

Usually the currents in the dots $\mathbf{j}_{j}(\mathbf{r})$ are defined by solving the linearized hydrodynamic equations for the averaged electron velocities. The solution of these equations leads to Ohm's law

$$
\mathbf{j}_{j}(\mathbf{r})=-\left.\overleftrightarrow{\sigma}^{(j)} \cdot \nabla \delta \varphi(\mathbf{r}, z)\right|_{z=0, a}
$$

In the presence of an external applied perpendicular magnetic field, the components of the conductivity tensor $\stackrel{\leftrightarrow}{\sigma}^{(j)}$ in polar coordinates are given by

$$
\begin{aligned}
\sigma_{r r}^{(j)} & =\sigma_{\theta \theta}^{(j)}=\frac{i \omega e^{2} \rho_{j}(r)}{m\left(\omega^{2}-\omega_{c}^{2}\right)}, \\
\sigma_{r \theta}^{(j)} & =\sigma_{\theta r}^{(j)}=\frac{\omega_{c} e^{2} \rho_{j}(r)}{m\left(\omega^{2}-\omega_{c}^{2}\right)} .
\end{aligned}
$$

Here the symbol $\omega_{c}=e B / m c$ stands for the electron cyclotron frequency, and $\omega$ is the frequency of the external perturbation, i.e. the far-infrared radiation. Poisson's equation together with Eqs. (2.6) and (2.7) compose the basic set for the coupled dots, from which the excitation spectrum will be obtained.

In order to calculate the power absorption spectrum the same equation set has to be used but with the single replacement $\delta \varphi \rightarrow \delta \varphi+\varphi^{e x t}$, where the external field potential $\varphi^{e x t}$ is added. Then the frequency dependent power absorption spectrum is calculated in the standard way using the following expression

$$
P(\omega)=-\frac{\omega}{2 \pi} \int_{0}^{2 \pi / \omega} d t \sum_{j=1}^{2} \int d^{2} r \mathbf{j}_{j} \cdot \nabla \varphi^{e x t} .
$$

\section{EQUILIBRIUM PROPERTIES}

The first step in the static problem is obtained by replacing Poisson's equation (2.1) by the homogeneous Laplace equation which is solved with the following boundary conditions for the potential in the dots

$$
\left.\frac{\partial \varphi}{\partial z}\right|_{z=0}=\frac{2 \pi e}{\epsilon} \rho_{1},\left.\quad \frac{\partial \varphi}{\partial z}\right|_{z=a}=\frac{2 \pi e}{\epsilon} \rho_{2} .
$$

As was shown in Refs. 1215 for the case of a single dot the most simple way to treat this static problem is to use the oblate spheroidal coordinate system centered in the dot, which is defined as follows: $x=\sqrt{\left(\sigma^{2}+1\right)\left(1-\tau^{2}\right)} \cos \theta, y=\sqrt{\left(\sigma^{2}+1\right)\left(1-\tau^{2}\right)} \sin \theta, z=$ $\sigma \tau$. In this coordinate system the disk corresponds to the region $\sigma=0,0 \leq \tau \leq 1$. The advantage of this coordinate system is that the variables in Laplace's equation can be separated and the solutions can be expressed as a linear superposition of the potential 
harmonics $10 P_{j}^{|m|}(\tau) Q_{j}^{|m|}(i \sigma)$, with $j \geq|m| . \quad P_{j}^{|m|}$ and $Q_{j}^{|m|}$ are the associated Legendre polynomials of the first and the second kind, respectively. Now the static problem is reduced to the solution of an algebraic set of equations for the expansion coefficients which follows from the equilibrium conditions.

We shall use a similar approach in our coupled dot problem. In order to have the most simple expressions for the main part of the electron-electron interaction, namely the electron-electron interaction in the same dot, we introduce two coordinate systems each centered in the corresponding dot. We rescale both coordinate systems, replacing $r \rightarrow r R_{i}$, and introduce the oblate spheroidal coordinates in each of the dots, respectively. Next, we divide the potential into two parts $\varphi=\varphi_{1}+\varphi_{2}$, each created by the electron density in the corresponding dot, and introduce dimensionless variables by performing the following transformations

$$
\rho_{j}(r) \longrightarrow \rho_{j}(r) \rho_{j}^{(0)} \sqrt{1-r^{2}}, \quad \varphi_{j} \longrightarrow \frac{k_{j} R_{j}^{2}}{|e|} \varphi_{j} .
$$

By this transformation we explicitly took out the non-analyticity of the density function at the dot edge which is inherent in the case of a soft confining potential. 12 If we choose the scaling factors $\rho_{j}^{(0)}=2 \epsilon k_{j} R_{j} / \pi^{2} e^{2}$, introduce the dimensionless numbers of electrons in the dots $n_{j}=3 N_{j} / 2 \pi R_{j}^{2} \rho_{j}^{(0)}$, chemical potentials $v_{j}=\mu_{j} / k_{j} R_{j}^{2}$, and ratios $\gamma=R_{2} / R_{1}$ and $\kappa=k_{2} / k_{1}$, we transform our static Eqs. (2.2), (3.1), and (2.4) into the following set of dimensionless equations

$$
\begin{aligned}
& r^{2} / 2+\varphi_{1}^{\text {dot }}(r)+\gamma^{2} \kappa \varphi_{2}^{\text {out }}(\tilde{r})=v_{1}, \\
& r^{2} / 2+\frac{1}{\gamma^{2} \kappa} \varphi_{1}^{\text {out }}(\tilde{r})+\varphi_{2}^{\text {dot }}(r)=v_{2}, \\
& \left.\frac{\partial \varphi_{j}}{\partial \sigma}\right|_{\sigma=0}=\frac{2 \tau^{2}}{\pi} \rho_{j}(r), \\
& \int_{0}^{1} d r r \sqrt{1-r^{2}} \rho_{j}(r)=\int_{0}^{1} d \tau \tau^{2} \rho_{j}(r)=\frac{n_{j}}{3} .
\end{aligned}
$$

Here the symbol $\tilde{r}$ stands for the coordinate in one dot calculated in the coordinate system of the other dot. The superscripts indicate whether the potential is created by the electron density in the same dot $\left({ }^{d o t}\right)$ or the electron density outside it $\left({ }^{\text {out }}\right)$.

Laplace's equation must not be included into the set of equations because, as is discussed above, its solutions are already known 12 , 16 and can be presented as

$$
\varphi_{j}^{\text {out }}(r)=\sum_{l} C_{l}^{(j)} P_{2 l}(\tau) \frac{Q_{2 l}(i \sigma)}{Q_{2 l}(0)},
$$

where due to the circular symmetry of the equilibrium state only the even potential harmonics with $m=0$ have to be taken into account. This general expression can be used for the potential outside the dot. Inside the $\operatorname{dot}(\sigma=0)$ the potential can be presented in a more simple form

$$
\varphi_{j}^{d o t}(r)=\sum_{l} C_{l}^{(j)} P_{2 l}(\tau) .
$$


The electron density in the dot can also be presented as an expansion in Legendre polynomials

$$
\rho_{j}(r)=\frac{1}{\tau^{2}} \sum_{l} n_{l}^{(j)} P_{2 l}(\tau)
$$

Here, the additional factor $\tau^{2}$ is included in order to simplify the boundary condition (3.30) which now leads to the relation $n_{l}^{(j)}=L_{2 l}^{0} C_{l}^{(j)}$ between the coefficients in both expansions (3.5) and (3.6), with

$$
L_{l}^{|m|}=\frac{\pi}{2} \frac{\Gamma((l+|m|) / 2+1) \Gamma((l-|m|) / 2+1)}{\Gamma((l+|m|+1) / 2)) \Gamma((l-|m|+1) / 2)}
$$

where the symbol $\Gamma$ stands for the Gamma function. Inserting expansions (3.4), (3.5) and (3.6) into Eqs. 3.3a, 3.3b), multiplying them by $P_{2 l}(\tau)$, integrating over $\tau$ and using the Legendre polynomial orthogonality condition

$$
\int_{0}^{1} d \tau P_{l}^{m}(\tau) P_{l^{\prime}}^{m}(\tau)=\frac{\delta_{l, l^{\prime}}}{2 l+1} \frac{(l+m) !}{(l-m) !}
$$

we obtain the following set of linear equations

$$
\begin{aligned}
C_{l}^{(1)}+\kappa \sum_{n} T_{l n}(1 / \gamma, d / \gamma) C_{n}^{(2)} & =\left(v_{1}-\frac{1}{3}\right) \delta_{l, 0}+\frac{1}{3} \delta_{l, 2}, \\
C_{l}^{(2)}+\frac{1}{\kappa} \sum_{n} T_{l n}(\gamma, d) C_{n}^{(1)} & =\left(v_{2}-\frac{1}{3}\right) \delta_{l, 0}+\frac{1}{3} \delta_{l, 2} .
\end{aligned}
$$

Here the symbol $T_{l n}$ stands for the overlap integral of the potential harmonics created by the electron densities in the different dots

$$
T_{l n}(\gamma, d)=\frac{(2 l+1)}{\gamma^{2}} \int_{0}^{1} d \tau P_{2 l}(\tau) P_{2 n}(\tilde{\tau}) \frac{Q_{2 n}(i \tilde{\sigma})}{Q_{2 n}(0)}
$$

with $\tilde{\tau}=\left\{\left[\sqrt{q^{2}+4 d^{2}}-q\right] / 2\right\}^{1 / 2}, \quad \tilde{\sigma}=\left\{\left[\sqrt{q^{2}+4 d^{2}}+q\right] / 2\right\}^{1 / 2}$ and $q=\gamma^{2}\left(1-\tau^{2}\right)+d^{2}-1$. In the above expressions we used the dimensionless distance between the two dots $d=a / R_{1}$. Eqs. (3.9) should be supplemented by two more conditions (as was shown in Ref. 12)

$$
\sum_{l} L_{2 l} P_{2 l}(0) C_{l}^{(j)}=0
$$

which guarantees the non singular behavior of the density functions at the dot edge $\tau=0$.

Conditions (3.11) together with Eqs.(3.9) form a set of coupled linear algebraic equations for the coefficients $v_{j}$ and $C_{l}^{(j)}$, which is equivalent to a nonhomogeneous matrix equation for the vector $X=\left\{v_{1}, C_{0}^{(1)}, \cdots, v_{2}, C_{0}^{(2)}, \cdots\right\}$. Only in the case of well separated dots $(a \rightarrow \infty)$ an analytical solution can be found. In that case we obtain $v_{1}=v_{2}=n_{1}=n_{2}=1$ which corresponds to the single parabolic dot solution.12 For the general case we solved the problem numerically by means of $Q R$-decomposition of the truncated matrix.17 We restricted our consideration to 9 potential harmonics for each dot which was sufficient to 
obtain an accuracy better that $0.1 \%$. We should note that in the case of different confining potentials $\kappa \neq 1$ or with an applied bias $e V \neq 0$ the numerical solution can not be found straightforwardly because of the unknown ratio $\gamma$ of the dot radii. We found $\gamma$ by solving the boundary condition (2.3) iteratively. After the ratio of the dot radii is found all quantities of interest can be calculated just using the scaling factors introduced in Sec. II], namely,

$$
\begin{array}{r}
N_{1}=\frac{n_{1}}{n_{1}+\gamma^{3} \kappa n_{2}} N, \quad N_{2}=\gamma^{3} \kappa \frac{n_{2}}{n_{1}} N_{1}, \\
R_{1}=\frac{1}{\left(n_{1}+\gamma^{3} \kappa n_{2}\right)^{(1 / 3)}} R_{0}, \quad R_{2}=\gamma R_{1}, \\
\rho_{1}^{(0)}=\frac{1}{\left(n_{1}+\gamma^{3} \kappa n_{2}\right)^{(1 / 3)}} \rho_{0}, \quad \rho_{2}^{(0)}=\gamma \kappa \rho_{1}^{(0)} .
\end{array}
$$

Here the symbol $N=N_{1}+N_{2}$ stands for the total number of electrons in boths dots, and two scaling factors $-R_{0}=\left(3 \pi e^{2} N / 4 \epsilon k_{1}\right)^{1 / 3}$ and $\rho_{0}=3 N / 2 \pi R_{0}^{2}$ - are used. The first one corresponds to the equilibrium radius of the first dot when all electrons are situated in this dot.

The numerical results illustrating the equilibrium electron densities in the dots are shown in Figs. 2 and 3 . The density profiles for equivalent coupled dots $(\kappa=1)$ are given in Fig. 2 for various distances $a$ between the two dots. In the case when there is no external bias $\left(\mu_{1}=\mu_{2}\right)$ both dots have the same density of electrons $\left(N_{1}=N_{2}=N / 2\right)$. In the limiting case of small distances between the dots the result reduces to the single parabolic dot with

density profile $\rho_{i} \sim \sqrt{1-r^{2} / R_{0}^{2}}$. In the opposite case of large interdot distances we again have two parabolic dots where in the above density functions the radius is scaled by $2^{1 / 3}$. For intermediate distances, however, the density profile differs from that of a single parabolic dot. This is illustrated in Fig. 2 by the thick dashed-dotted curve which shows the parabolic dot density with the same dot size and the same total number of electrons as those for the coupled dots with interdot distance $a / R_{0}=0.3$. The dependence of the dot size, $R_{i}$, on the interdot separation is shown in the inset of Fig. 2. With decreasing interdot separation the size of the dots increases and the electron density profile is more spread out. In Fig. 3 the density profiles for the case of different confining potential strengths $\left(\kappa=2, \mu_{1}=\mu_{2}\right)$ are given for the same values of the interdot distances as in Fig. 2. Notice that now the density profiles differ essentially from the single parabolic dot solution. In the case of small interdot distances even a ring like distribution can be obtained when the electrons in the dot with the weakest confinement are pushed outside. The above ring like distribution effect can be even more reinforced by applying an external bias. Notice also that with decreasing interdot distance electrons from dot 2 are moved into dot 1 in order to keep the chemical potential the same (see inset of Fig. 3(b)). The inset of Fig. 3(a) shows the dependence of the radius of the two dots on the distance between the two dots.

\section{MAGNETOPLASMA EXCITATIONS}

To solve the dynamic equations (2.1), (2.6) and (2.7) we use the same oblate spheroidal coordinate technique as in the static case which was described in previous section. Restricting ourselves only to those modes which can be excited in the dipole approximation we use the following expansions for the harmonic deviations of the electron density 


$$
\delta \rho_{j}(\mathbf{r})=\frac{1}{\tau^{2}} \sum_{l} g_{l}^{(j)} P_{2 l+1}^{1}(\tau) e^{i \alpha \theta}
$$

the potential inside the dot

$$
\delta \varphi_{j}^{d o t}(\mathbf{r})=\sum_{l} c_{l}^{(j)} P_{2 l+1}^{1}(\tau) e^{i \alpha \theta}
$$

and the potential outside the dot

$$
\delta \varphi_{j}^{\text {out }}(\mathbf{r})=\sum_{l} c_{l}^{(j)} P_{2 l+1}^{1}(\tau) \frac{Q_{2 l+1}^{1}(i \sigma)}{Q_{2 l+1}^{1}(0)} e^{i \alpha \theta} .
$$

The symbol $\alpha= \pm 1$ is the angular momentum of the excited harmonics actually indicating the polarization of the circularly polarized electric field. The above electron density and potential harmonics obey the same boundary conditions in the dots as given by Eq. (3.30) which leads to the relation $g_{l}^{(i)}=L_{2 l+1}^{1} c_{l}^{(i)}$. We will use the same dimensionless variables as in previous static case scaling additionally the frequencies $\omega \rightarrow \omega \cdot \omega_{1}$ and $\omega_{c} \rightarrow \omega_{c} \cdot \omega_{1}$. In those dimensionless variables the continuity equation (2.6) becomes

$$
\begin{aligned}
& \omega\left(\omega^{2}-\omega_{c}^{2}\right) \tau \delta \rho_{1}-\nabla \cdot\left(\stackrel{\leftrightarrow}{s}^{(1)} \cdot \nabla\left(\delta \varphi_{1}^{\text {dot }}+\delta \varphi_{2}^{\text {out }}\right)\right)=0, \\
& \frac{\omega}{\kappa}\left(\omega^{2}-\omega_{c}^{2}\right) \tau \delta \rho_{2}-\nabla \cdot\left(\stackrel{\leftrightarrow}{s}^{(2)} \cdot \nabla\left(\delta \varphi_{1}^{\text {out }}+\delta \varphi_{2}^{\text {dot }}\right)\right)=0,
\end{aligned}
$$

where for the sake of convenience we introduced the tensor $\stackrel{\leftrightarrow}{s}^{(i)}$, which is proportional to the static densities, with the following components

$$
s_{r r}^{(i)}=s_{\theta \theta}^{(i)}=\omega \tau \rho_{i}(\tau), \quad s_{r \theta}^{(i)}=-s_{\theta r}^{(i)}=-i \omega_{c} \tau \rho_{i}(\tau)
$$

Now inserting expansions (4.1), (4.2) and (4.3) into Eqs. 4.4a, 4.4b), multiplying the obtained expressions by $\tau P_{l}^{1}(\tau) \exp (-i \alpha \theta)$, integrating over $\theta$ and $\tau$, and using the orthogonality condition (3.8) we obtain the following set of equations for the coefficients $c_{l}^{(i)}$

$$
\begin{aligned}
& \omega\left(\omega^{2}-\omega_{c}^{2}\right) \frac{2(l+1)(2 l+1)}{4 l+3} L_{2 l+1}^{1} c_{l}^{(1)}-\sum_{n} V_{l n}^{(1)} c_{n}^{(1)}-\kappa \sum_{n} W_{l n}^{(2)}(1 / \gamma, d / \gamma) c_{n}^{(2)}=0, \\
& \frac{\omega}{\kappa}\left(\omega^{2}-\omega_{c}^{2}\right) \frac{2(l+1)(2 l+1)}{4 l+3} L_{2 l+1}^{1} c_{l}^{(2)}-\frac{1}{\kappa} \sum_{n} W_{l n}^{(1}(\gamma, d) c_{n}^{(1)}-\sum_{n} V_{l n}^{(2)} c_{n}^{(2)}=0,
\end{aligned}
$$

which is cast into a homogeneous matrix equation

$$
\mathcal{M}(\omega) X=0,
$$

for the vector $X=\left\{c_{0}^{(1)}, c_{1}^{(1)}, \cdots, c_{0}^{(2)}, c_{1}^{(2)}, \cdots\right\}$. The dynamic matrix $\mathcal{M}$ is composed of the overlap integrals of the potential components belonging to the same dot

$$
\begin{aligned}
V_{l n}^{(i)}= & \frac{1}{2 \pi} \int_{0}^{2 \pi} d \theta \int_{0}^{1} d \tau \tau P_{2 l+1}^{1}(\tau) e^{-i \alpha \theta} \nabla \cdot\left(\stackrel{\leftrightarrow}{s}^{(i)} \cdot \nabla P_{2 n+1}^{1}(\tau) e^{i \alpha \theta}\right) \\
= & \int_{0}^{1} d \tau P_{2 l+1}^{1}(\tau)\left\{-\frac{s_{r r}^{(i)}}{\tau}[n(n+1)-1]\right. \\
& \left.+\left(1-\tau^{2}\right)\left[\frac{d}{d \tau}\left(\frac{s_{r r}^{(i)}}{\tau}\right)\right] \frac{\partial}{\partial \tau}-i \alpha\left(\frac{d s_{r \theta}^{(i)}}{d \tau}\right)\right\} P_{2 n+1}^{1}(\tau) .
\end{aligned}
$$


and to the different dots

$$
\begin{aligned}
& W_{l n}^{(i)}(\gamma, d)=\int_{0}^{1} d \tau P_{2 n+1}^{1}(\tilde{\tau}) \frac{Q_{2 n+1}^{1}(i \tilde{\sigma})}{Q_{2 n+1}^{1}(0)}\left\{-\frac{s_{r r}^{(i)}}{\tau}[l(l+1)-1]\right. \\
& \left.+\left(1-\tau^{2}\right)\left[\frac{d}{d \tau}\left(\frac{s_{r r}^{(i)}}{\tau}\right)\right] \frac{\partial}{\partial \tau}+\left(\frac{d s_{r \theta}^{(i)}}{d \tau}\right)\right\} P_{2 l+1}^{1}(\tau) .
\end{aligned}
$$

The functions $\tilde{\tau}, \tilde{\sigma}$ and $q$ are defined as in the stationary case.

The magnetoplasma excitation frequencies $\omega_{j}$ are obtained as solutions of the following equation

$$
\operatorname{det} \mathcal{M}(\omega)=0
$$

The matrix determinant was calculated numerically by means of LU-decomposition 17 truncating the matrix up to 7 potential components for each dot which guaranteed the same accuracy as in the equilibrium case.

\section{POWER ABSORPTION}

We restrict our consideration to the case of weak dissipation when the magnetoplasma excitation spectrum can be most easily revealed. In that particular case the power absorption can be calculated using the same set of dynamic equations (4.4a, 4.4b) but now with the external electric field added. Our system is circular symmetric and therefore it is most convenient to consider the circularly polarized electric field $\mathbf{E}^{\mathrm{ext}}=E_{0}\left(\mathbf{e}_{x}+i \alpha \mathbf{e}_{y}\right) \exp (i \omega t)$

with the corresponding external potential amplitude $\varphi^{\operatorname{ext}}(\mathbf{r})=-E_{0}(x+i \alpha y)$. Now inserting the external potential into the set of dynamic equations (4.4a, 4.4b), performing the same procedure as before, and scaling the vector $X \rightarrow X \sqrt{2 \pi}|e| E_{0} / k_{1} R_{1}$ we arrive at the following nonhomogeneous matrix equation

$$
\mathcal{M}(\tilde{\omega}) X=B
$$

Here, the vector $B=\left\{b_{0}^{(1)}, b_{1}^{(1)}, \cdots, b_{0}^{(2)}, b_{1}^{(2)}, \cdots\right\}$ is composed of the following components

$$
b_{l}^{(i)}=2(l+1)(2 l+1)\left(\omega+\alpha \omega_{c}\right) \sum_{l^{\prime}} S_{l^{\prime}, l^{\prime}} n_{l^{\prime}}^{(i)} \cdot\left\{\begin{array}{cc}
1, & i=1, \\
1 / \kappa \gamma, & i=2,
\end{array}\right.
$$

and

$$
S_{l^{\prime}, l}=\int_{0}^{1} \frac{d \tau}{\tau} P_{2 l^{\prime}}(\tau) P_{2 l+1}(\tau)
$$

is an overlap integral of even and odd Legendre polynomials.

Besides, in order to take some small dissipation into account we applied the standard procedure making the following frequency replacement $\omega \rightarrow \tilde{\omega}=\omega+i \eta$. The symbol $\eta$ represents a phenomenological inverse electron relaxation time, and takes into account the 
internal dissipation within the electron fluid in the dot. According to expression (2.9) the power absorption can be presented as

$$
P(\omega)=-\omega P_{0} \operatorname{Im} L_{1}^{1}\left\{c_{0}^{(1)}+\kappa \gamma^{4} c_{0}^{(2)}\right\},
$$

where we have introduced the absorption power scaling factor $P_{0}=4 \epsilon E_{0}^{2} R_{1}^{3} \omega_{1} / 3 \pi$. Formally, the dimensionless absorption power can be expressed in matrix form

$$
p(\omega)=P(\omega) / P_{0}=-\operatorname{Im}\{T \cdot X\},
$$

where the vector $T=\left\{\omega L_{1}^{1}, 0, \cdots, 0, \omega \kappa \gamma^{4} L_{1}^{1}, 0, \cdots, 0\right\}$ has only two non zero components.

Equation (5.1) and the power absorption (5.5) was solved numerically using the $S V D$ (singular value decomposition) 17 of the dynamical matrix

$$
\mathcal{M}(\tilde{\omega})=\mathcal{U} \Lambda(\tilde{\omega}) \mathcal{V}^{T}
$$

where $\mathcal{U}$ and $\mathcal{V}$ are unitary matrices (superscript ${ }^{T}$ stands for transposed matrix) and $\Lambda$ is the diagonal matrix composed of the eigenvalues $\lambda_{i}(\tilde{\omega})$ of the dynamical matrix $\mathcal{M}$. Due to the decomposition (5.6) the solution of equation (5.1) can be presented as

$$
X=\mathcal{V} \Lambda^{-1}(\tilde{\omega}) \mathcal{U}^{T} B
$$

Inserting it into expression (5.5) we obtain the general expression for the power absorption

$$
p(\omega)=-\operatorname{Im}\left\{T \cdot \mathcal{V} \Lambda^{-1}(\tilde{\omega}) \mathcal{U}^{T} B\right\} .
$$

This expression can be further simplified taking into account that we are considering the case of weak dissipation $(\eta \rightarrow 0)$ only. In that case the power absorption consists of narrow Lorentzians located at the magnetoplasma excitation frequencies $\omega_{j}$. According to Eq. (4.11) the corresponding conditions $\lambda_{i}\left(\omega_{i}\right)=0$ should be fulfilled. This enables us to use the following expansion

$$
\lambda_{i}(\tilde{\omega})=\lambda_{i}^{\prime}\left(\omega_{i}\right)\left(\omega-\omega_{i}+i \eta\right) .
$$

Inserting it into expression (5.8) gives the following final expression for the power absorption

$$
p(\omega)=-\operatorname{Im} \sum_{i} \frac{\left(T \cdot V_{i}\right)\left(U_{i}^{T} \cdot B\right)}{\lambda_{i}^{\prime}\left(\omega_{i}\right)\left(\omega-\omega_{i}+i \eta\right)}=\sum_{i} \frac{\eta f_{i}}{\left(\omega-\omega_{i}\right)^{2}+\eta^{2}},
$$

where we introduced the coefficients

$$
f_{i}=\operatorname{Re} \frac{\left(T \cdot V_{i}\right)\left(U_{i}^{T} \cdot B\right)}{\lambda_{i}^{\prime}\left(\omega_{i}\right)}
$$

and the symbol $V_{i}$ stands for the $i$-th vector-column of matrix $\mathcal{V}$, and the symbol $U_{i}^{T}$ for the $i$-th vector-row of the transposed matrix $\mathcal{U}^{T}$. Note that we neglected the imaginary part of the right hand side of expression (5.11), because for weak dissipation it only leads to a negligible eigenfrequency shift. For the same reason the other quantities (like $\left.\mathcal{U}, \mathcal{V}, \lambda^{\prime}\right)$ should be estimated at $\omega=\omega_{i}$.

In the case of weak dissipation the quantities (5.11) together with the mode frequencies $\omega_{i}$ characterise the power absorption. By analogy to quantum mechanical problems they can be referred to as the classical oscillator strengths. As is shown in Appendix A these oscillator strengths obey a general sum rule like their quantum mechanical counterparts, indicating that the interaction variation in the system leads to the redistribution of power absorption among various vibration modes while the total power absorption remains unchanged. 


\section{RESULTS AND DISCUSSION}

For the case of equivalent dots $\kappa=1$ and $\mu_{1}=\mu_{2}$ the eigenfrequencies and oscillator strengths are given in Fig. 1 . The frequencies in the limiting case of $a \rightarrow \infty$ are shown in Fig. 4(a) as function of the applied magnetic field $\left(\omega_{c}\right)$. The eigenfrequencies for clockwise circular polarization $(\alpha=1)$ are shown by solid curves while the dashed curves indicate the eigenfrequencies for the opposite polarization $(\alpha=-1)$. In the limit case $a \rightarrow \infty$ of decoupled dots one finds that $W_{l n}^{(i)}=0$ and $V_{l n}^{(i)}$ is a diagonal matrix (see Eqs. (4.9) and (4.10)). Then the eigenmodes are the basisfunctions $P_{2 l+1}^{1}(\tau)$ and the spectrum branches can be uniquely labeled with the numbers $(l, \alpha= \pm 1)$. In this limit the eigenfrequencies are twofold degenerate and coincide with the single parabolic dot frequencies presented in Ref. 12. In the low frequency region a large set of branches are found which correspond to the edge modes. They are not resolved and shown up as a shaded area. In Fig. 四(b) the spectrum for an interdot separation of $a / R_{0}=0.5$ is presented. For the sake of convenience we still label the branches by the previous $l$-values in order to show the correspondance of the branch to its counterpart in the $a \rightarrow \infty$ limiting case. In this case in fact several $l$-values are mixed. Note that when the distance between the layers decreases the degeneracy is lifted and all branches are split into two and are shifted down in frequency except for the two branches depicted by the thick curves which correspond to the center of mass motion of the whole system of electrons in both dots. Only the latter two frequencies have non zero oscillator strengths which do not depend on the interdot distance and are shown in Fig. 四(c). This is in accordance with Kohn's theorem which can be easily generalized to our vertically aligned two dot system for the case of identical dots, i.e. $\kappa=1$. The decrease of the eigenfrequencies with decreasing interdot distance is a consequence of the increase of the repulsive interaction of the electrons in the different dots and is discussed in more detail in Appendix B.

The case of nonequivalent dots is illustrated in Figs. 5 and 6 where the results for $\kappa=2$ and $\mu_{1}=\mu_{2}$ are presented. The frequencies for the $a \rightarrow \infty$ limit are shown in Fig. 5(a), and the corresponding oscillator strengths are shown in Fig. 5(b). In this case the system consists of two noninteracting parabolic dots where the frequencies are scaled

by the factor $\sqrt{\kappa}=\sqrt{2}$. The branches are labeled with the corresponding $l$-value. The non-zero oscillator strenghts are those which corresponds to the center of mass motions of the electron systems in the separate dots. Note that the oscillator strengths obey the sum rule that the sum of the oscillator strengths equals 1 independent of $\omega_{c}$. The redistribution of the oscillator strengths between the separate dot branches actually reflects the electron number redistribution between the dots (see Fig. 3). It is also evident that those spectrum branches which are closer to the cyclotron resonance branch $\left(\omega=\omega_{c}\right)$ have essentially larger oscillator strengths.

The spectrum branches and the oscillator strengths change fundamentally when nonequivalent dots are put closer to each other as is shown in Fig. 6. Now all the branches are shifted down in frequency and non-zero oscillator strengths corresponding to other then the center of mass motion branches start to appear. For instance, in Fig. 6(b) the oscillator strengths corresponding to the frequency branches $(l=1)$ are non-zero as shown by the curves labeled 5 and 6 . The reason is that for nonequivalent dots the electron density distributions are far away from the parabolic dot distribution (see, Fig. 3), and Kohn's theorem 
is no longer valid. Note also from Fig. 6(a) that the frequency shift to lower frequencies is much more pronounced as compared to the case of equivalent dots (Fig. 田(b)). The above frequency shift can be even more amplified when an external bias is applied. This is illustrated in Fig. 7 where the results for $\kappa=2$ and $\mu_{1}-\mu_{2}=-0.2$ are given. From Fig. 7(a) we see that now the $(l=1)$ branches start to interfere with the $(l=0)$ ones. Although there is still no intersection with the branches of the same polarization the branch $(l=1, \alpha=1)$ approaches the cyclotron resonance line $\left(\omega=\omega_{c}\right)$ so closely that the oscillator strength (see Fig. $7(\mathrm{~b}))$ is transferred from the main branch $(l=0, \alpha=1)$.

We would like to note that in the above case of nonequivalent dots when the system no longer obeys Kohn's theorem the edge modes have small but non-zero oscillator strengths. In order to reveal those weak modes we performed a more accurate dynamic matrix diagonalisation for the case $\mu_{1}=\mu_{2}, a / R_{0}=0.5$ and $\kappa=2$ by restricting ourselves to the $l=0$ and $l=1$ components for each dot. The results for the edge modes (curves 9 and 10) together with the oscillator strengths for the upper $l=1$ modes (curves 7 and 8) are given in Fig. 8. We see that the oscillator strengths become very small for spectrum branches which are substantially different from the above mentioned $\omega=\omega_{c}$.

\section{CONCLUSIONS}

In this paper, we have studied the magnetoplasma excitations in two vertically coupled electron dots in the dipole approximation. We used the classical hydrodynamic approach which is adequate in the limit of a large number of electrons. The confinement potential was taken parabolic in both dots. We calculated the equilibrium electron density distribution as function of the interdot distance. As function of the magnetic field the frequency spectrum and the corresponding oscillator strengths are calculated.

In the case of equivalent dots, i.e. both dots have the same parabolic confinement strength, the equilibrium density distribution is similar to the single dot parabolic one, and due to the generalized Kohn's theorem only the center of mass motion can be excited.

If both dots have a different parabolic confinement the electron densities can differ appreciably from the parabolic case. Even ring like electron distributions are found. Due to the fact that both dots have different confinement potentials Kohn's theorem is no longer valid and other modes then the center of mass motions can be excited. The largest oscillator strengths are found for the modes closest to the cyclotron frequency. If an external bias potential is applied the oscillator strengths of other then the center of mass modes are even more enhanced. Crossover phenomena can be observed in this case. For nonequivalent dots, even the edge modes have small but non-zero oscillator strengths.

\section{ACKNOWLEDGMENTS}

B.P. is an Aspirant and F.M.P. a Research Director of the Flemish Science Foundation (FWO-Vlaanderen). This work is partially supported by a NATO-linkage Collaborative Research Grant, the FWO-Vlaanderen and the 'Interuniversity Poles of Attraction Program - Belgian State, Prime Minister's Office - Federal Office for Scientific, Technical and Cultural Affairs'. 


\section{APPENDIX A: SUM RULE FOR THE CLASSICAL OSCILLATOR STRENGTHS}

In this Appendix we present the definition of the classical oscillator strength for a general linear mechanical system with weak dissipation and we proof a sum rule for these oscillator strengths. We consider a mechanical system interacting with an external force which can be described by the following matrix equation

$$
\left(\frac{d}{d t}-\mathcal{L}\right) X(t)=A f(t) .
$$

Here symbol $X$ stands for the vector composed of the system variables: coordinates, velocities etc. $\mathcal{L}$ is the dynamical matrix describing the mechanical system, $A$ is the unit length vector $\left(A^{2}=1\right)$ characterizing the application of the external force, and the scalar function $f(t)$ stands for the external force itself. The power absorption is usually calculated for a periodic driving force

$$
f(t)=f(\omega) \cos (\omega t)=\operatorname{Re}\left\{f e^{i \omega t}\right\} .
$$

In that case the averaged power absorption is

$$
\begin{aligned}
P(\omega) & =\frac{\omega}{2 \pi} \int_{0}^{2 \pi / \omega} d t P(t)=\frac{\omega}{2 \pi} \int_{0}^{2 \pi / \omega} d t f(t)\left(A^{T} \cdot X(t)\right) \\
& =\frac{1}{2} \operatorname{Re}\left\{f^{*}(\omega)\left(A^{T} \cdot X(\omega)\right)\right\},
\end{aligned}
$$

where the coordinate vector Fourier component obeys the following equation

$$
(i \omega-\mathcal{L}) X(\omega)=A f .
$$

Note that our set of dynamic equations (4.6a, 4.6b) can be easily reduced to this type of equation by just introducing additional variables $Y=\omega X, Z=\omega Y$ and constructing the resulting vector $\hat{X}=\{X, Y, Z\}$ of all those variables.

Now we use the following dynamical matrix representation

$$
\mathcal{L}=\mathcal{U} \mathcal{D U}^{-1}
$$

where $\mathcal{D}$ is the diagonal matrix composed of the eigenvalues $i \omega_{i}$ corresponding to the system eigenfrequencies. Using that expansion and the substitution $\omega \rightarrow \tilde{\omega}=\omega+i \eta$ we obtain the solution of equation (A4)

$$
\begin{aligned}
X(\omega) & =\mathcal{U}(i \tilde{\omega}-\mathcal{D})^{-1} \mathcal{U}^{-1} A f \\
& =f \sum_{i=1}^{n} U_{i}\left\{i\left(\omega-\omega_{i}\right)-\eta\right\}^{-1}\left(U_{i}^{-1} \cdot A\right),
\end{aligned}
$$

and the expression for the power absorption

$$
\begin{aligned}
P(\omega) & =\frac{1}{2} f^{2} \operatorname{Re} \sum_{i=1}^{n}\left(A^{T} \cdot U_{i}\right)\left\{i\left(\omega-\omega_{i}\right)-\eta\right\}^{-1}\left(U_{i}^{-1} \cdot A\right) \\
& =\frac{1}{2} f^{2} \sum_{i=1}^{n} \frac{f_{i} \eta}{\left(\omega-\omega_{i}\right)^{2}+\eta^{2}},
\end{aligned}
$$


where

$$
f_{i}=\operatorname{Re}\left\{\left(A^{T} \cdot U_{i}\right)\left(U_{i}^{-1} \cdot A\right)\right\},
$$

is the oscillator strength. Like in Sec. $\square$ symbol $U_{i}$ stands for the $i$-th vector-column of matrix $\mathcal{U}$, and the symbol $U_{i}^{-1}$ is the $i$-th vector-row of matrix $\mathcal{U}^{-1}$, and the term $\operatorname{Im}\left\{\left(A^{T} \cdot U_{i}\right)\left(U_{i}^{-1} \cdot A\right)\right\}$ is neglected because in the case of weak dissipation it gives a negligible eigenfrequency shift.

The oscillator strengths obey a useful sum rule which follows straightforwardly from definition (A8), namely,

$$
\begin{aligned}
\sum_{i=1}^{n} f_{i} & =\operatorname{Re} \sum_{i=1}^{n}\left\{\left(A^{T} \cdot U_{i}\right)\left(U_{i}^{-1} \cdot A\right)\right\} \\
& =\operatorname{Re}\left(A^{T} \cdot U U^{-1} \cdot A\right)=\left(A^{T} \cdot A\right)=1 .
\end{aligned}
$$

We obtained a relation which shows that the sum of the oscillator strengths does not depend on the properties of the dynamical system. Note we omitted symbol Re in the last equation (A9) because the classical equations of type (A1) are always formulated with real coefficients.

The obtained sum rule has a very simple physical meaning. It is just related to the total power absorption in the case of the impact external force $f(t)=A \delta(t)$. Actually, in that case calculating the total power absorption

$$
P=\int_{-\infty}^{\infty} d t f(t)\left(A^{T} \cdot X(t)\right)=\int_{-\infty}^{\infty} d t \delta(t)\left(A^{T} \cdot X(t)\right)
$$

we only need to know the solution in the limit of small time values. That solution can be obtained just neglecting the dynamical matrix $\mathcal{L}$ in equation (A1) as compared with the time derivative. So, the solution reads

$$
X(t)=A \int_{-\infty}^{t} d t \delta(t)
$$

and the total power absorption

$$
P=\int_{-\infty}^{\infty} d t f(t)\left(A^{T} \cdot X(t)\right)=\left(A^{T} \cdot A\right) \int_{-\infty}^{\infty} d t \delta(t) \int_{-\infty}^{t} d t^{\prime} \delta\left(t^{\prime}\right)=\frac{1}{2} \sum_{i=1}^{n} f_{i}
$$

coincides with half of the above defined sum. Thus, the total absorption power does not depend on the properties of the dynamical system, and that confirms that the sum of oscillator strengths is a universal quantity.

\section{APPENDIX B: TWO COUPLED OSCILLATORS WITH REPULSIVE INTERACTION}

In Sec. VI it was shown that all the spectrum branches show a general behavior, namely the frequencies decrease with decreasing interdot distance. Such behavior is caused by the repulsive interaction of the electrons belonging to different dots, and can be explained using the following simple model. We focus our attention to the center of mass motions of the 
electrons of the seperate dots. In the case the dots are decoupled $(a \rightarrow \infty)$ those motions can be treated as two independent oscillators in a magnetic field perpendicular to the plane of motion. When the dots are put closer to each other, say, to some distance $a \gg R_{i}$, the interdot electron interaction can be approximated by

$$
\frac{e^{2}}{\sqrt{\left(\mathbf{r}_{1}-\mathbf{r}_{2}\right)^{2}+a^{2}}} \approx \frac{e^{2}}{a}-\frac{e^{2}}{2 a^{3}}\left(\mathbf{r}_{1}-\mathbf{r}_{2}\right)^{2} .
$$

Here the coordinate $\mathbf{r}_{i}$ corresponds to the lateral center of mass motion of the electrons in dot $i$. The first term in the above expression gives the total energy shift while the second one should be inserted into the equations describing the system oscillations. Thus our model system consists of two harmonic oscillators with a repulsive harmonic interaction in a magnetic field. If also an external clockwise circularly polarized electric field is applied, the equations read

$$
\begin{aligned}
& m \ddot{\mathbf{r}}_{1}+k_{1} \mathbf{r}_{1}+\frac{e}{c} \dot{\mathbf{r}}_{1} \times \mathbf{B}-\frac{e^{2}}{a^{3}}\left(\mathbf{r}_{1}-\mathbf{r}_{2}\right)=e N_{1} E_{0}\left(\cos (\omega t) \mathbf{e}_{\mathbf{x}}-\sin (\omega t) \mathbf{e}_{\mathbf{y}}\right), \\
& m \ddot{\mathbf{r}}_{2}+k_{2} \mathbf{r}_{1}+\frac{e}{c} \dot{\mathbf{r}}_{2} \times \mathbf{B}+\frac{e^{2}}{a^{3}}\left(\mathbf{r}_{1}-\mathbf{r}_{2}\right)=e N_{2} E_{0}\left(\cos (\omega t) \mathbf{e}_{\mathbf{x}}-\sin (\omega t) \mathbf{e}_{\mathbf{y}}\right),
\end{aligned}
$$

where $n_{1}$ and $N_{2}$ are the total number of electrons in each dot, and $E_{0}$ is the amplitude of the applied electric field. This linear set of equations can be solved by introducing the complex variables $z_{i}=x_{i}+i y_{i}$ which leads to the following set of equations

$$
\begin{aligned}
& \ddot{z}_{1}+i \omega_{c} \dot{z}_{1}+\omega_{1}^{2} z_{1}-\Omega_{0}^{2}\left(z_{1}-z_{2}\right)=\frac{e N_{1} E_{0}}{m} e^{-i \omega t}, \\
& \ddot{z}_{2}+i \omega_{c} \dot{z}_{2}+\omega_{2}^{2} z_{2}+\Omega_{0}^{2}\left(z_{1}-z_{2}\right)=\frac{e N_{2} E_{0}}{m} e^{-i \omega t} .
\end{aligned}
$$

Here $\Omega_{0}^{2}=e^{2} / m a^{3}$. Now inserting $z_{i} \sim \exp (-i \omega t)$ into the homogenious version of the above expressions and mapping the four obtained eigenfrequencies for both polarizations onto the positive frequencies we have

$$
\omega^{1,2,3,4}=\sqrt{\left(\frac{\omega_{c}}{2}\right)^{2}+\frac{\omega_{1}^{2}+\omega_{2}^{2}}{2}-\Omega_{0}^{2} \pm \sqrt{\left(\frac{\omega_{1}^{2}-\omega_{2}^{2}}{2}\right)^{2}+\Omega_{0}^{4}}} \pm \frac{\omega_{c}}{2} .
$$

It is easily seen that the derivative

$$
\left.\frac{d \omega^{1,2,3,4}}{d \Omega_{0}^{2}}\right|_{\Omega_{0}=0}=\frac{-1}{\sqrt{\left(\frac{\omega_{c}}{2}\right)^{2}+\frac{\omega_{1}^{2}+\omega_{2}^{2}}{2} \pm\left(\frac{\omega_{1}^{2}-\omega_{2}^{2}}{2}\right)}},
$$

is always negative what confirms that the interdot interaction of electrons causes a negative frequency shift, and thus, illustrates the previously obtained negative shift of the spectrum branches with decreasing interdot distance.

The power absorption for this model system can also easily be calculated. It reads

$$
P(\omega)=\frac{1}{2 m} e\left(N_{1}+N_{2}\right) E_{0} \sum_{i=1}^{4} \frac{f_{i} \eta}{\left(\omega-\omega_{i}\right)^{2}+\eta^{2}},
$$


where the oscillator strengths are given by

$$
f_{i}=\frac{\left(\omega^{i}\right)^{3}+\omega_{c}\left(\omega^{i}\right)^{2}+\left(\Omega_{0}^{2}-\frac{N_{1}}{N_{1}+N_{2}}\left(\omega_{2}^{2}-\Omega_{0}^{2}\right)-\frac{N_{2}}{N_{1}+N_{2}}\left(\omega_{1}^{2}-\Omega_{0}^{2}\right)\right) \omega^{i}}{\prod_{j \neq i}\left(\omega^{i}-\omega^{j}\right)} .
$$

It can be checked straightforwardly that the oscillator strengths obey the sum rule $\sum_{i=1}^{4} f_{i}=$ 1. They show the correct qualitative behavior as function of the cyclotron frequency as shown for the coupled dots in Sect. VI. 


\section{REFERENCES}

* Electronic mail: bpartoen@uia.ua.ac.be

- Permanent address: Semiconductor Physics Institute, Goštauto 11, 2600 Vilnius, Lithuania. Electronic mail: matulis@pub.osf.lt

- Electronic mail: peeters@uia.ua.ac.be

${ }^{1}$ N. F. Johnson, J. Phys.: Condens. Matter 7, 965 (1995).

${ }^{2}$ W. Kohn, Phys. Rev. 123, 1242 (1961); L. Brey, N. F. Johnson, and B. I. Halperin, Phys. Rev. B 40, 10647 (1989); P. A. Maksym and T. Chakraborty, Phys. Rev. Lett. 65, 108 (1990); F. M. Peeters, Phys. Rev. B 42, 1468 (1990).

${ }^{3}$ J. J. Palacios and P. Hawrylak, Phys. Rev. B 51, 1769 (1995).

${ }^{4}$ S. C. Benjamin and N. F. Johnson, Phys. Rev. B 51, 14733 (1995).

${ }^{5}$ H. Imamura, P. A. Maksym, and H. Aoki, Phys. Rev. B 53, 12613 (1996).

${ }^{6}$ B. Partoens, V. A. Schweigert, and F. M. Peeters, Phys. Rev. Lett. 79, 3990 (1997).

${ }^{7}$ G. Goldoni and F. M. Peeters, Phys. Rev. B 53, 4591 (1996); Europhys. Lett. 37, 293 (1997).

${ }^{8}$ D. G. Austin, T. Honda, and S. Tarucha, Jpn. Appl. Phys. 36, 1667 (1997); Semicond. Sci. Technol. 12, 631 (1997).

${ }^{9}$ D. Leonard, M. Krishnamurthy, C. M. Reaves, S. P. Denbaars, and P. M. Petroff, Appl. Phys. Lett. 63, 3203 (1993); R. Nötzel, T. Fukui, and H. Hasegawa, Appl. Phys. Lett. 65, 2854 (1994); J. Oshinowo, M. Nishioka, S. Ishida, and Y. Arakawa, Appl. Phys. Lett. 65, 1421 (1994).

${ }^{10}$ N. Qureshi, J.S. Scott, S.J. Allen, M. Reddy, M. Rodwell, I. Tanaka, T. Noda, I. Kamiya, J. Kone, and H. Sakaki, presented at the workshop on: Dynamics in Quantum Structures far from Equilibrium (Santa Barbara, CA, 11-12 July 1997).

${ }^{11}$ G. S. Solomon, J. A. Trezza, A. f. Marshall, and J. S. Harris, Jr., Phys. Rev. Lett. 76, 952 (1996).

${ }^{12}$ Z. L. Ye and E. Zaremba, Phys. Rev. B 50, 17217 (1994).

${ }^{13}$ S. S. Nazin and V. B. Shikin, Sov. J. Low. Temp. Phys. 15, 127 (1989) [Fiz. Nizk. Temp. 15, $227(1989)]$.

${ }^{14}$ V. Shikin, S. Nazin, D. Heitmann, and T. Demel, Phys. Rev. B 43, 11903 (1991).

${ }^{15}$ S. Nazin, K. Tevosyan, and V. Shikin, Surf. Sci. 263, 351 (1992).

${ }^{16}$ Methods of Theoretical Physics, P. M. Morse and H. Feshbach (McGraw-Hill Book Company, Tokyo, 1953) chpt. 10.

${ }^{17}$ Numerical Recipes in C, W. H. Press, S. A. Teukolsky, W. T. Vetterling, and B. P. Flannery (Cambridge University Press, 1994) chpt. 2. 


\section{FIGURES}

FIG. 1. The layout of the structure.

FIG. 2. The equilibrium electron density profiles for the system with $\kappa=1$ and $\mu_{1}=\mu_{2}$ for different values of $a / R_{0}$. The inset shows the radius of the dots as function of $a / R_{0}$.

FIG. 3. The equilibrium electron density profiles for the system with $\kappa=2$ and $\mu_{1}=\mu_{2}$ for the same values of $a / R_{0}$ as in Fig. 2. The inset in (a) shows the radii of the dots, the inset in (b) the number of electrons in dot 1 as function of $a / R_{0}$.

FIG. 4. The frequency spectrum for the system with $\kappa=1$. The limiting case $a / R_{0}=\infty$ is shown in (a) where the branches are labeled by the corresponding $l$ value. The case $a / R_{0}=0.5$ is shown in (b). The solid lines correspond to the $(\alpha=1)$ branches, the dotted lines to the $(\alpha=-1)$ ones. The branches that can be excited in the dipole approximation are indicated by thick curves. The corresponding oscillator strengths are shown in (c).

FIG. 5. The frequency spectrum and the oscillator strength for the system with $\kappa=2$ and $\mu_{1}=\mu_{2}$ in the limit $a / R_{0}=\infty$. The notation is the same as in Fig. 4 . The correspondence between the spectrum branches and the oscillator strengths are indicated by the numbers 1-4.

FIG. 6. The frequency spectrum and the oscillator strengths for the system with $\kappa=2$ and $\mu_{1}=\mu_{2}$ for the interdot distance $a / R_{0}=0.5$. The numbers give the corresponding frequency branches and oscillator strengths. Only 6 oscillator strengths are shown.

FIG. 7. The frequency spectrum and the oscillator strength for the system with $\kappa=2$, $a / R_{0}=0.3$ and with an external applied bias potential $\mu_{1}-\mu_{2}=-0.2$. The numbers give the corresponding frequency branches and oscillator strengths. Only 7 oscillator strengths are shown.

FIG. 8. The edge modes and the oscillator strengths for the system with $\kappa=2$ and $\mu_{1}=\mu_{2}$. The interdot distance is $a / R_{0}=0.5$. The series (3.5) and (4.2) are approximated by only the first two terms. The numbers give the corresponding frequency branches and oscillator strengths. 
Figure 1

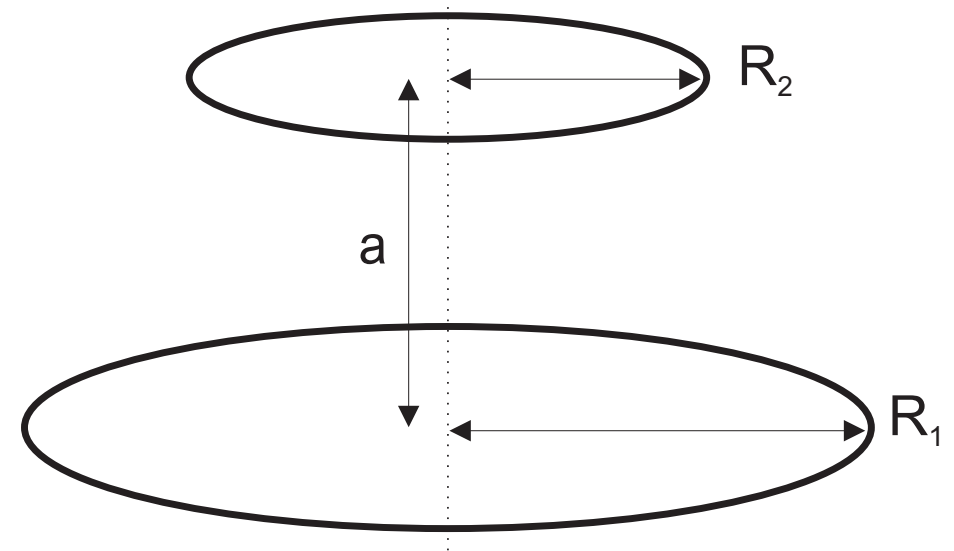


Figure 2

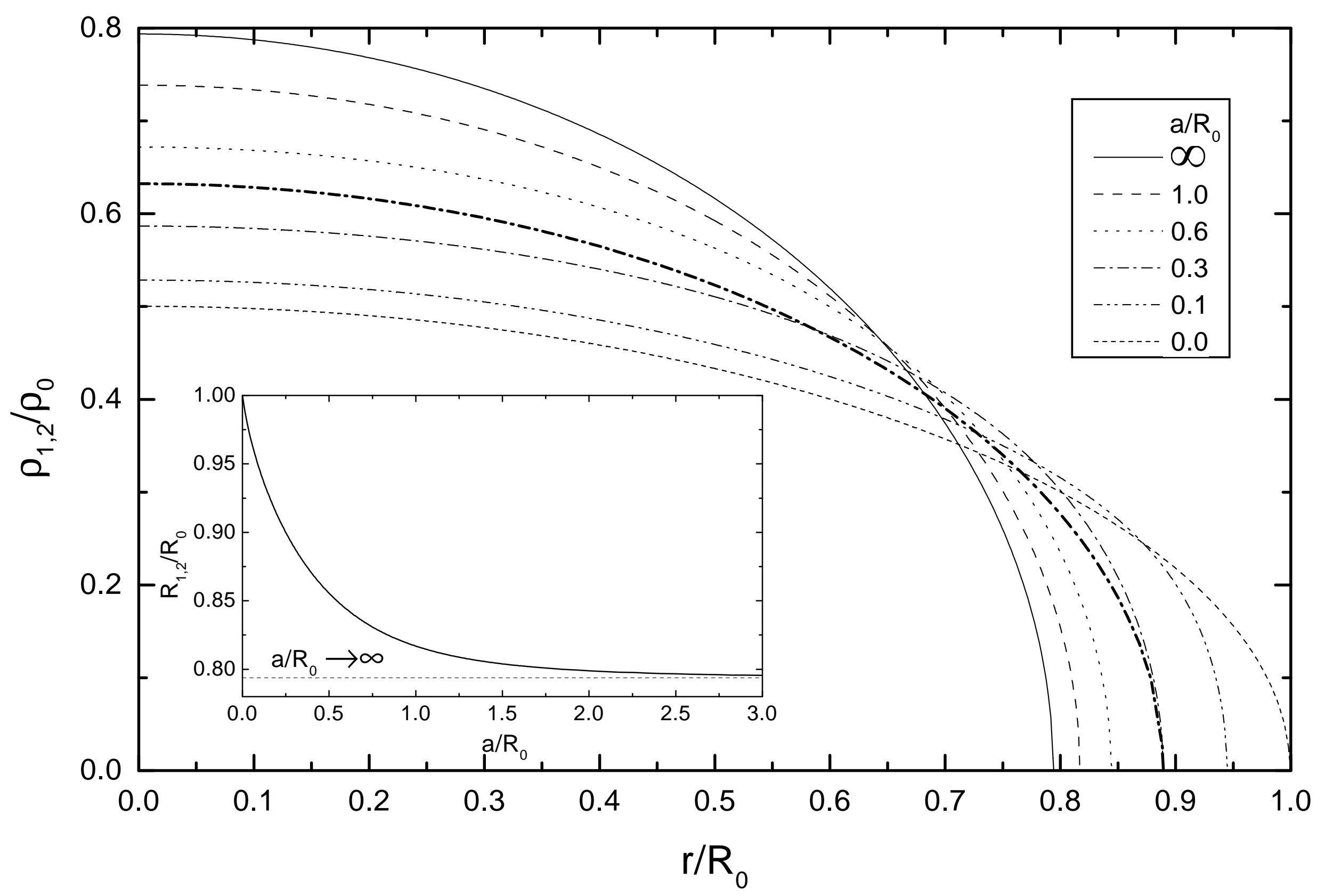


Figure 3
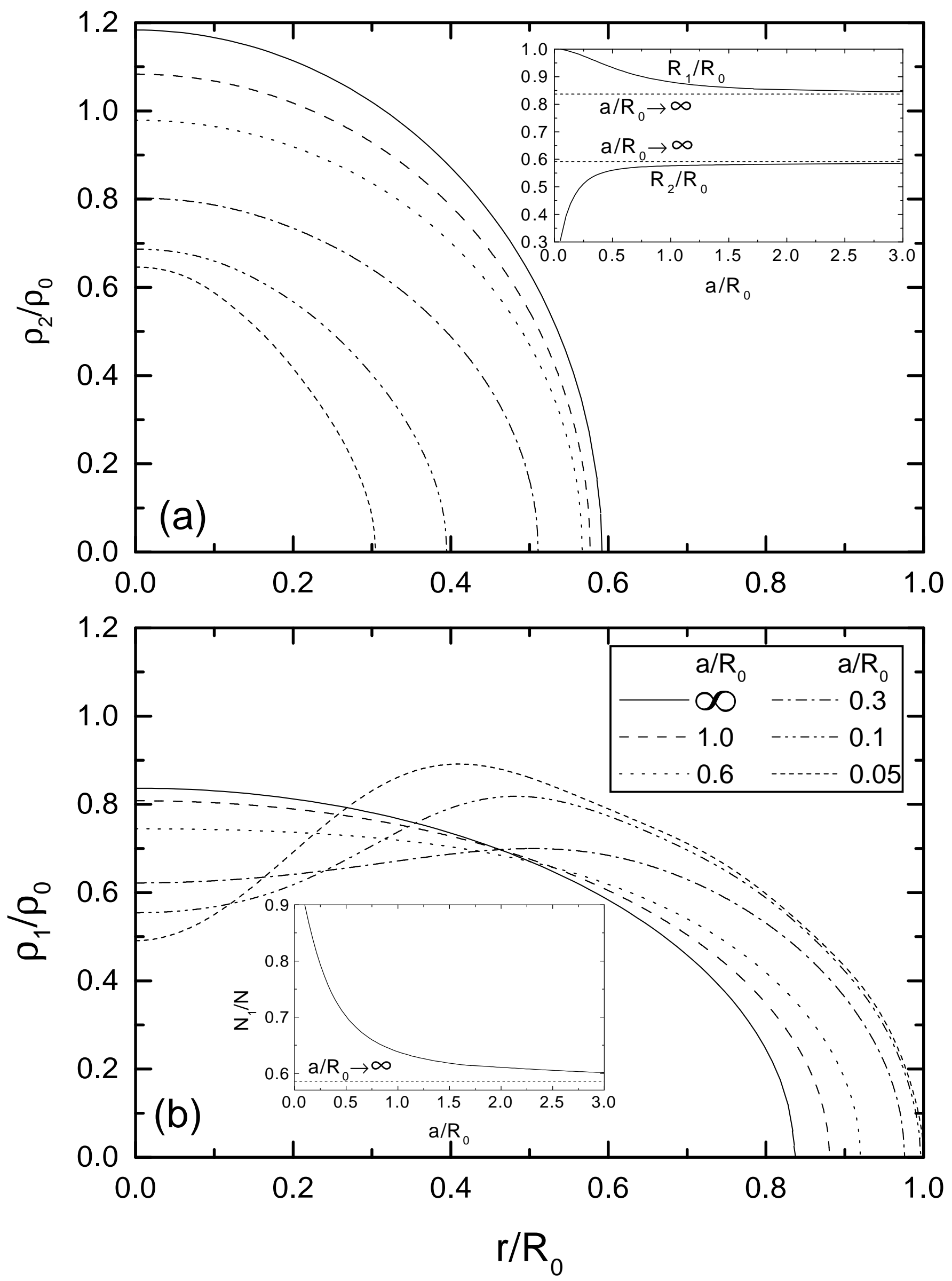
Figure 4
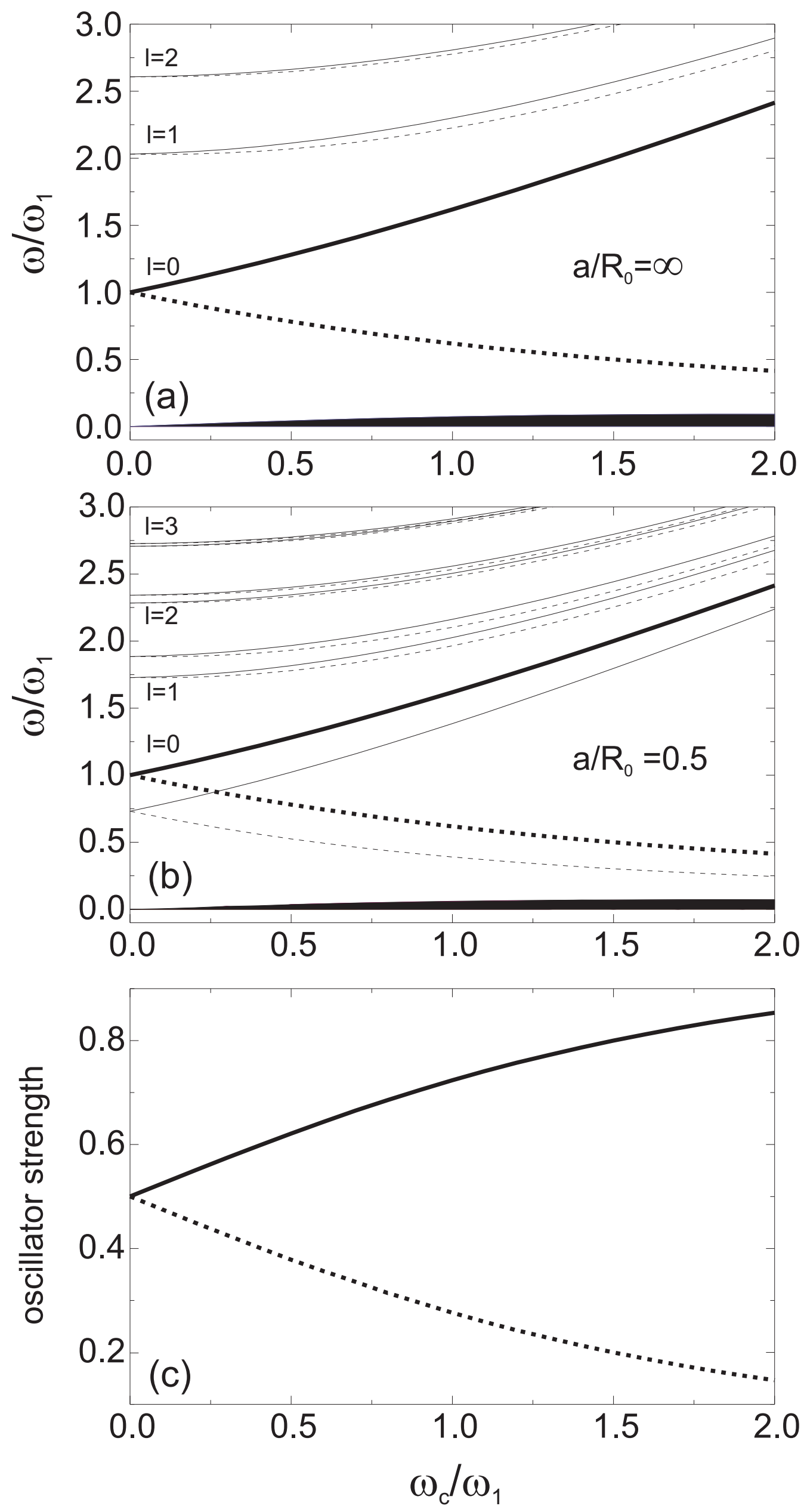
Figure 5
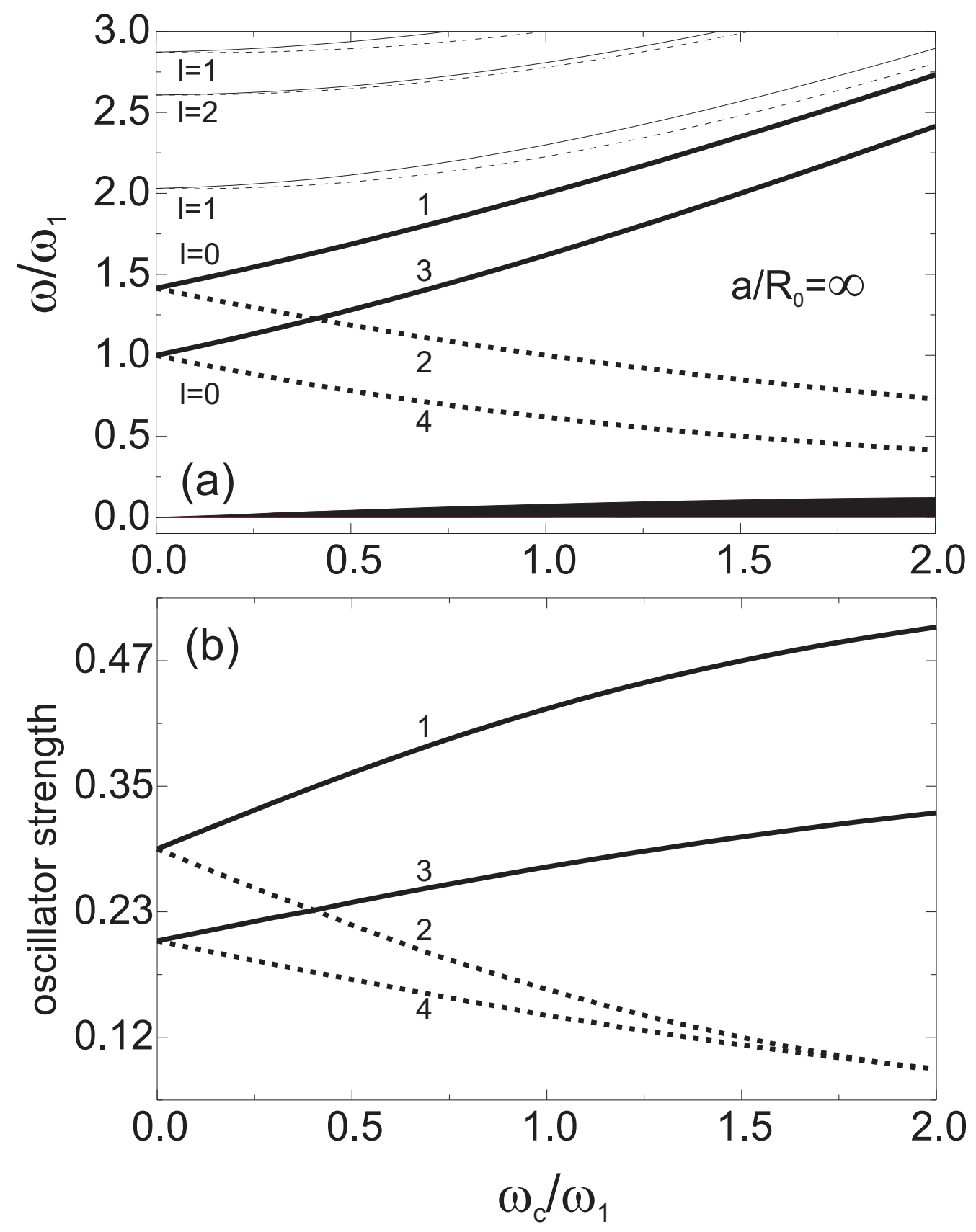
Figure 6
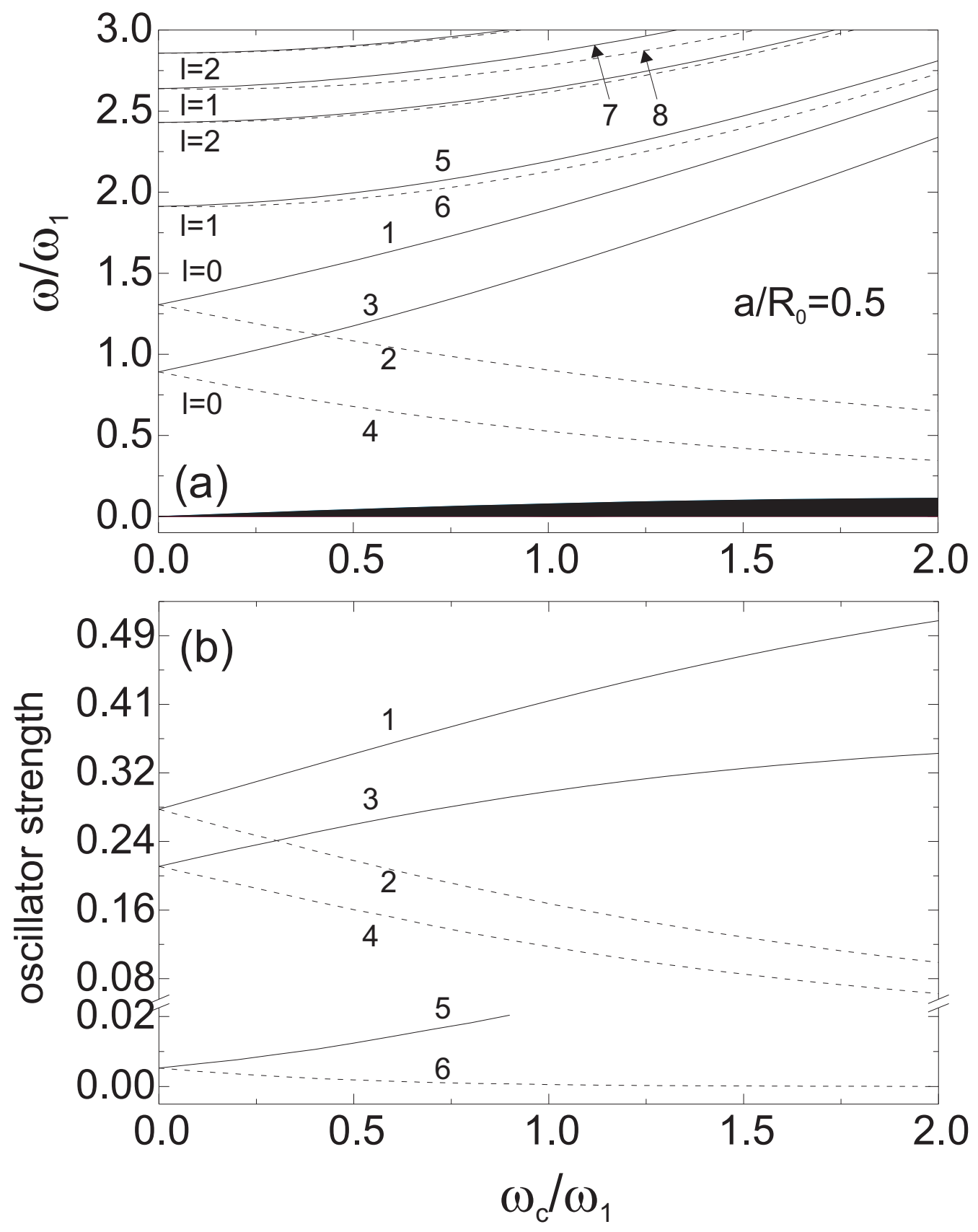
Figure 7
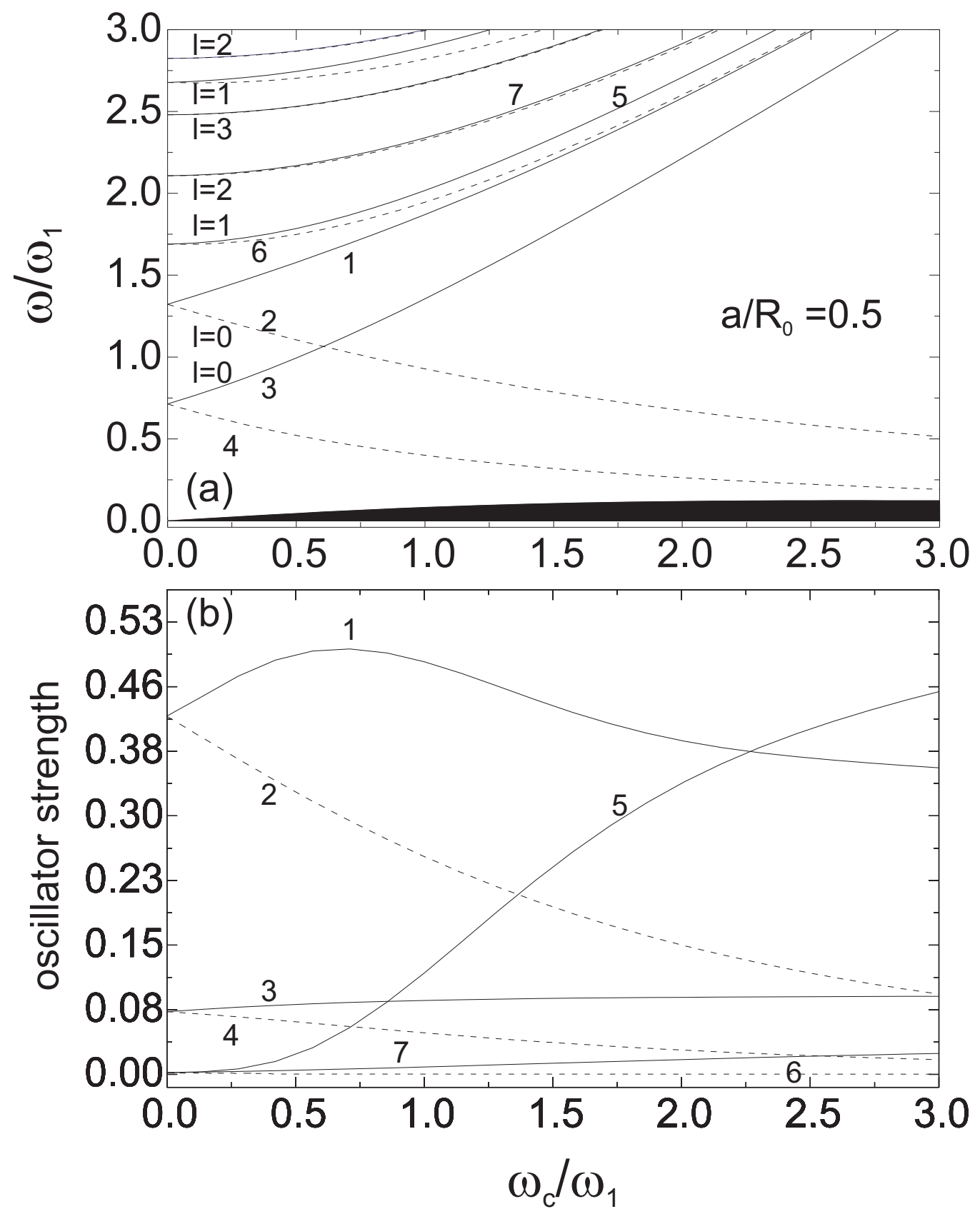
Figure 8
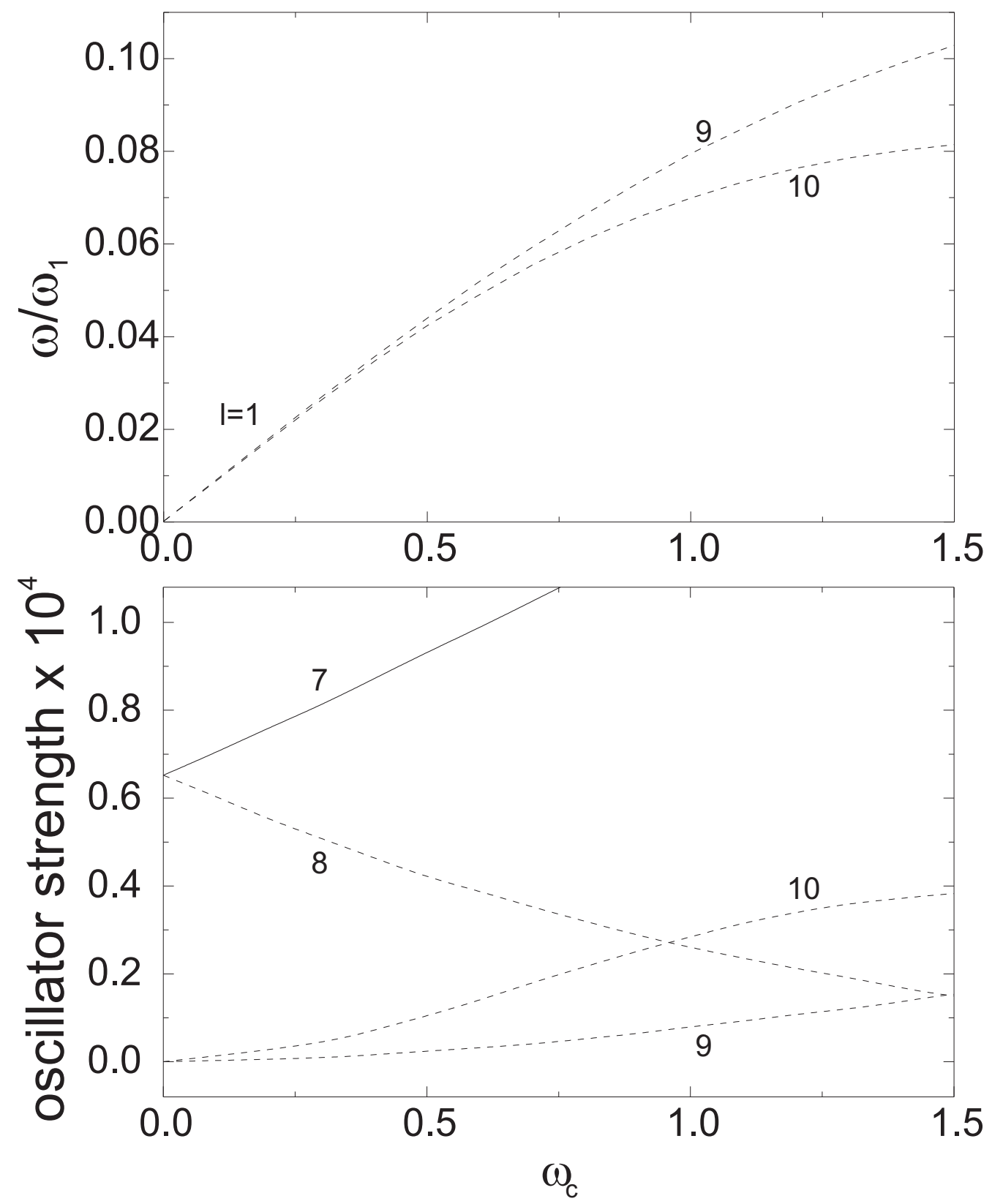